\title{
A Neural Network Approach to the Recognition of the $K$ Distribution Shape Parameter associated with Sea Clutter
}

\section{Una Aproximación Neuronal al Reconocimiento del Parámetro de Forma de la Distribución K asociada a Clutter Marino}

\author{
José Raúl Machado Fernández. \\ Instituto Superior Politécnico José Antonio Echeverría \\ m4ch4do@hispavista.com \\ Briam García Delgado \\ Instituto Superior Politécnico José Antonio Echeverría \\ m4ch4do@hispavista.com \\ Alejandro Machado Gil \\ Instituto Superior Politécnico José Antonio Echeverría \\ m4ch4do@hispavista.com
}

Aceptado: 8 de agosto 2016

\begin{abstract}
The main problem faced today by sea radars is the elimination of clutter, which is an undesirable contribution that appears mixed with the target information. The unwanted signal is produced by the echo caused by the reflections of the primary emission at the sea surface. One of the most popular probability distributions in clutter modeling is the $\mathrm{K}$ distribution. Helpful in efficient detectors desing, a system able to recognize the shape parameter of the $\mathrm{K}$ distribution, knowing a priori the value of the scale parameter, is proposed. The result is appropriate for real time operating conditions as it is based on a neural networks approximation in the pattern recognition role.
\end{abstract}

Keywords

Sea Clutter, Artificial Neural Networks, K Distribution, Estimation of Distribution Parameters, machine learning, statistical modeling.

\section{Resumen}

El principal problema que enfrentan los radares marinos es la eliminación del clutter que es una señal indeseable que aparece mezclada con la información del blanco. El origen de este aporte interferente está en el eco resultante del rebote de la emisión primaria en la superficie marina. Una de las distribuciones probabilísticas más populares en la modelación del clutter es la 
distribución K. Beneficioso en el diseño de detectores eficientes, es propuesto un sistema capaz de reconocer el parámetro de forma de la distribución K conociendo de antemano el valor del parámetro de escala. El resultado es aplicable a condiciones de operación en tiempo real, pues se basa en una aproximación de redes neuronales artificiales en rol de reconocimiento de patrones.

\section{Palabras clave}

Clutter Marino, Redes Neuronales Artificiales, Distribución K, Estimación de Parámetros de Distribuciones Probabilísticas, aprendizaje por computadora, modelación estadística. 


\section{INTRODUCTION}

Sea clutter is an undesirable signal that appears mixed with target information when radars operate in coastal or offshore environments (1). While some authors have used semi-deterministic chaotic models (2), the stochastic representation is the most popular when it comes to clutter modeling (3-7). It consist basically in the gathering of data on the behavior of sea echoes and its subsequent association with known distributions seeking a high level of similarity. The application of distributions is usually based on statistical results taken from amplitude measurements, since they are the most common in radars worldwide.

There are many distributions that may be used to model sea clutter, but the most widely accepted are the Log-Normal (5), the Weibull (8) and the K (9-11), defined by a recent research as classical sea representing distributions (12). The $\mathrm{K}$ distribution is one of the most important within the group according to the opinion of several authors (13-15), because it offers an explanation for the representation of the phenomenon; the rest of the distributions also match sea measurements, but they do not have a logical explanation for this to happen.

\subsection{Motivation and objectives}

Using the $\mathrm{K}$ distribution for clutter representation, the authors seek to design an artificial neural network able to recognize the shape parameter from histograms. The scale parameter, closely related to the mean of the distribution, is assumed to be known a priori.

If the goal is met, a system for clutter state estimation will be created with two important applications. First, a significant improvement in target detection will be achieved allowing the operation under the knowledge of the temporal and spatial behavior of the undesired signal; traditional systems that implement less efficient adaptive techniques assume no knowledge about the sea state (16). Secondly, clutter irregularities, which indicate the presence of a target or anomaly such as fish gatherings or oil spills, will become identifiable $(17,18)$.

\subsection{Contributions}

Authors build a system able to identify the value of the $\mathrm{K}$ shape parameter in the range between 0.1 and 16 , which was taken from recent investigations $(3,19)$. Using the MATLAB software, efforts to implement a single neural network did not bring suitable results, so it was decided to train several small individual networks to solve fractions of the problem. The final solution, which includes the interconnection of 12 individually trained neural networks, achieves the identification of the shape parameter with a high accuracy by reducing the uncertainty level to $6 \%$ of the initial range with a $98 \%$ of effectiveness.

\section{MATERIALS AND METHODS}

The Materials and Methods section is divided into three sub-sections. In the first of them a description of the $\mathrm{K}$ distribution, used for sea cluttermodeling, is given. The 
second sub-section is dedicated to describe the training set of the neural network using $\mathrm{K}$ distribution histograms as a base. Finally, the third sub-section presents the choice for the neural network internal configurable variables.

\subsection{K Distribution}

The $\mathrm{K}$ distribution is a compound model, which allows it to represent echoes from different states of the sea surface. It is primarily used for diffuse clutter measured at small grazing angles (20). Its Probability Density Function (PDF) is defined by the following expression (21).

$$
f(x)=\frac{4 c}{\Gamma(v)}(c x)^{v} K_{v-1}(2 c x)
$$

where

$\mathrm{c}$ is the scale parameter

$\mathrm{v}$ is the shape parameter

$\Gamma(\mathrm{v})$ is the gamma function

$\mathrm{K}(\mathrm{v}-1)$ is the modified Bessel function of the second kind and order $\mathrm{v}(20)$.
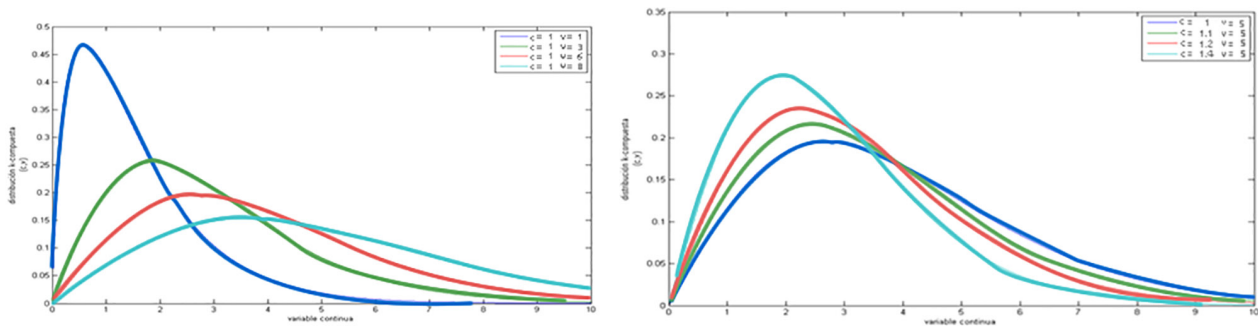

Figure 1. Draws from the K Probability Density Function.

Obtained in MATLAB, Fig. 1 shows the influence of the parameters on the characteristics of the $\mathrm{K}$ distribution plots. In the graph to the left, the shape parameter was maintained constant while the scale parameter was increased from 1 to 8. Also, the effect of the increase of the scale parameter is shown at the graph to the right where the shape parameter remained invariable.

An artificial neural network requires a training set in order to adapt its internal structure to the addressed problem (22). Therefore, the selection of the essentials features that describe the model to the network has a significant influence on the final result.

As it is evident in Fig. 1, a distribution is a family of PDF curves for which each combination of parameters results in a different draw. It should be noted that not all combinations allow sea clutter modeling but there are limits to the values for each parameter. After a review of the literature $(2,3,19,23)$, the authors selected the range 
from 0.5 to 5 for the scale parameter (c) and from 0.1 to 19.6 for the shape parameter (v), covering thus most radar situations.

\subsection{Selecting the training set}

Although studies aimed at identifying parameters have traditionally used the moments of the distribution as a distinctive feature (24), this time the authors preferred applying another approach that will prove to be effective. In order to describe the clutter, 200 samples were taken from each draw of the distribution by performing a uniform sampling.

Hence, the selected range of values for the K parameters is covered taking 200 samples while performing 0.045 shifts over the scale and 0.196 shifts over the shape parameter. Thus, 100 different values are included both for $\mathrm{c}$ and $\mathrm{v}$. The final result are 10000 groups of 200 samples, each representing a training unit of the neural network.

\subsection{Neural network design}

In addition to the training set, there are many other configurable parameters that may affect the performance of a neutral network (25). The MATLAB software chosen for the simulation allows the modification of many of them (26). The optimal algorithm consists on testing all possible configurations searching for the one that works best for the problem at hand. However, this alternative consumes a huge amount of time, which is why the authors preferred to follow recommendations from investigations that have successfully applied neural networks to solve radar problems (27-37).

Obeying the above analysis, Table 1 shows the selection of variables (or configuration parameters) for the neural network that will be trained. They were all maintained constant during the conducted experiments. The only exception of this rule is the variable "number of neurons in the hidden layer" that was left free to be optimized by trial-and-error essays.

Table 1. Selected Variables for Network Design.

\begin{tabular}{|c|c|}
\hline Design Variables & Selection \\
\hline Network Type & Feed Forward Network (Multilayer Perceptron) \\
\hline Training Function & $\begin{array}{c}\text { Scaled Conjugate Gradient Backpropagation (Online } \\
\text { Training) }\end{array}$ \\
\hline Number of Layers & 3 ( Input Layer - Hidden Layer - Output Layer) \\
\hline Transfer Function & $\begin{array}{c}\text { Hyperbolic Tangent Sigmoid (Hidden Layer and Output } \\
\text { Layer) }\end{array}$ \\
\hline Activation Order & Topological (Asynchronous Activation) \\
\hline Error Measurement & Mean Square Error \\
\hline Division of the Training Set & Training $70 \%$ - Validation $15 \%$ - Test $15 \%$ \\
\hline Order of Presentation of Samples & $\begin{array}{l}\text { The type of training is batch so the order of presentation } \\
\text { of samples has no influence }\end{array}$ \\
\hline
\end{tabular}




\section{RESULTS}

The Results section is devoted to the presentation of an artificial neural network able to identify accurately the value of the $\mathrm{K}$ distribution shape parameter. For a better understanding, it is divided into two sub-sections. The first one describes the initial training attempts that used a single network to solve the problem. After achieving negative results, the second sub-section proposes a structure consisting of several neural networks that achieves a high percentage of positive hits.

\subsection{First approach to training}

In order to identify the K shape parameter from samples taken from PDF histograms, the authors made several attempts to train an individual neural network. Even with the a priori knowledge of the scale parameter, it was not possible to obtain good results for hidden layer dimensions between 5 and 100 neurons. Similarly, the addition of multiple hidden layers did not improve the results significantly.

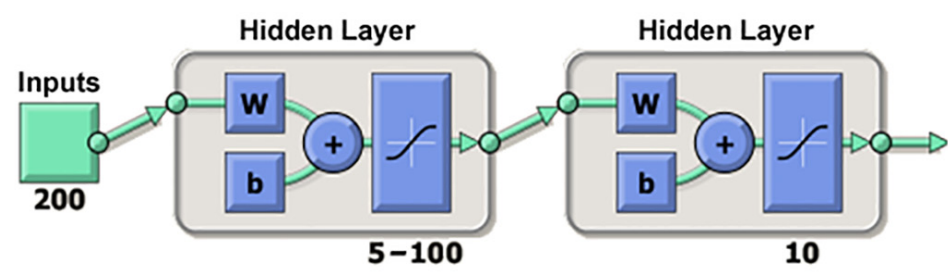

Figure 2. Diagram of the Neural Network for the First Approach.

Fig. 2 presents the structure of the initial network designed to find the K distribution shape parameter. This network included 10 outputs that were dedicated to indicate the range of values among which the parameter was located. The successes achieved with this network remained below $70 \%$, a totally unacceptable quantity. As it might be expected, after adding noise to the samples taken from histograms, the identification got worse falling to values close to $55 \%$.

\subsection{Second approach to training}

Although disappointing results were obtained, the authors persisted in the use of the neural model as a solution to the problem. In an innovative process, they designed what they called a Network of Networks. This new entity is composed by several neural networks that were trained independently to solve fractions of the main problem. The linked contribution of all networks gives the final solution to the estimation problem.

For training this Network of Networks, the 10000 groups of 200 samples were divided into five different sets of equal length. The new sets, with 2000 groups of samples each, covered the following parameter ranges: [1] 0.1 - 4.02 [2] 4.02 - 7.94 [3] 7.94 - 11.86 [4] 11.86 - 15.78, [5] 15.78 - 19 504. The selection of the boundaries 
between the intervals was not made at random, but the samples for which the network of the first approach made more mistakes were grouped into common sets. Thus, this decision represented an effort to separate a difficult problem into several easier ones.

Realizing that most of the first approach errors occurred in sets 3 and 5, the authors assumed that these samples presented to the network a problem of a different nature than their counterparts 1,2 and 4. Therefore, they prepared a neural network dedicated exclusively to the distinction between regions 1,2 and 4; and another one in charge of making the division between intervals 3 and 5. Promising results were obtained for these two networks.

Once ready these two specialized networks, the problem of distinguish when to apply one or the other emerged. Note that the system operates without any a priori knowledge about the value of the shape parameter of the samples. So, according to the division into five sets taking into account the range of the $\mathrm{K}$ parameters values, five new initial neural networks were added to the Network of Networks in order to choose which of the two specialized networks should be used. Each of the initial networks is ready to work with groups of samples from any $\mathrm{K}$ shape parameter set. The difference between them is that each one is trained to operate with only one of the five possible sets of the scale parameter, whose value is known in advance.

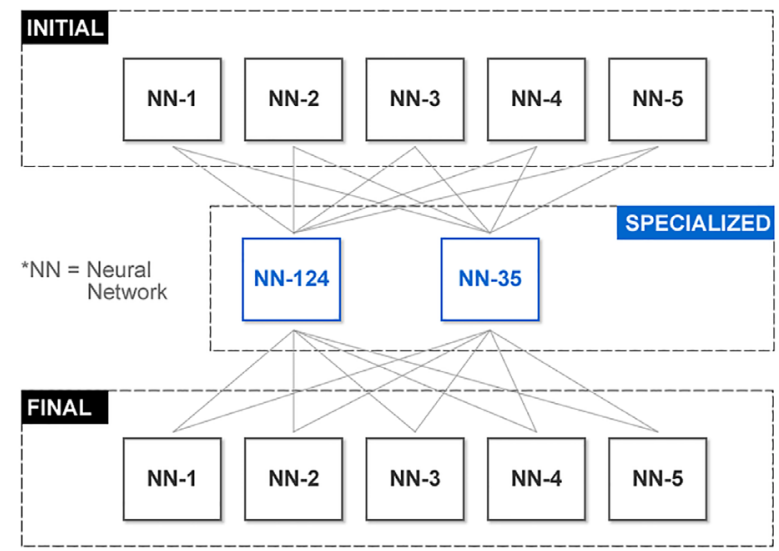

Figure 3. Diagram of the Network of Neural Networks for the Second Approach.

In addition to the seven above-mentioned networks, the authors decided to add five new final networks to narrow the range of the final result. Every one of them had five outputs, making a total of 25 . Only one of the 25 outputs is set to "one" in the normal operation of the Network of Networks indicating the range of values where the estimation decision is made. This final decision interval has a length of 0.784 units, which means that the maximum error that the network can make is 0.392 units. Fig. 3 shows the final diagram achieved with 12 neural networks, each with 25 or fewer neurons.

Once obtained satisfactory results with the Network of Networks, authors proceeded to train it with samples that presented imperfections, imitating the histograms formation process from a finite quantity of clutter samples. Thus, different Gaussian noise levels 
were added to the samples with Signal to Noise Ratio (SNR) of 50, 30, 20, 10 and 3 decibels $(\mathrm{dB})$. New Networks of Networks were trained with these modified sets and the performance of each was tested for the sets that were not use for its training. As a result, none of the created networks was able to work perfectly in all environments. The discussion of the selection of the best of them is left to the next section.

\section{DISCUSSION}

Table 2 shows the six Networks of Neural Networks trained for different levels of SNR. As it can be seen, they all exceeded $99 \%$ of accuracy when operating for sets with the same noise that the one that was used for their training. In addition, cases where the network was capable of generalizing its results for sets with lower noise level are highlighted, representing a desired behavior.

Table 2. Networks of Networks Performance when Facing Sets with Different SNR.

\begin{tabular}{ccccccc}
\hline \multicolumn{7}{c}{ Networks of Networks trained for different noise levels } \\
\hline $\begin{array}{c}\text { Signal to } \\
\text { Noise Ratio } \\
\text { of Test Sets }\end{array}$ & $\begin{array}{c}\text { Network of } \\
\text { Networks for } \\
\text { 3 dB SNR }\end{array}$ & $\begin{array}{c}\text { Network of } \\
\text { Networks for } \\
\text { 10 dB SNR }\end{array}$ & $\begin{array}{c}\text { Network of } \\
\text { Networks for } \\
\text { 20 dB SNR }\end{array}$ & $\begin{array}{c}\text { Network of } \\
\text { Networks for } \\
\text { 30 dB SNR }\end{array}$ & $\begin{array}{c}\text { Network of } \\
\text { Networks for } \\
50 \text { dB SNR }\end{array}$ & $\begin{array}{c}\text { Network of } \\
\text { Networks } \\
\text { without Noise }\end{array}$ \\
\hline $3 \mathrm{~dB}$ & $\mathbf{9 9 . 3}$ & 68.1 & 45.4 & 22.9 & 24.5 & 42.3 \\
$10 \mathrm{~dB}$ & 70.1 & $\mathbf{9 9 . 6}$ & 69.3 & 48.1 & 22.7 & 48.0 \\
$20 \mathrm{~dB}$ & 64.9 & 86.5 & $\mathbf{9 9 . 8}$ & 79.2 & 25.0 & 49.2 \\
$30 \mathrm{~dB}$ & 65.2 & 85.1 & 91.6 & $\mathbf{9 9 . 9}$ & 72.6 & 48.4 \\
$50 \mathrm{~dB}$ & 65.3 & 85.1 & 92.4 & 95.0 & $\mathbf{1 0 0}$ & 59.8 \\
$($ No noise) & 65.3 & 85.3 & 92.3 & 95.3 & 99.2 & $\mathbf{1 0 0}$ \\
\hline
\end{tabular}

If the level of signal to noise is known in advance, the network designed especially for it should be used. Otherwise, given the progressive deterioration of the hits percentage for sets with lower noise level that the one used in the training, the Network of Networks trained for 20 decibels is the best suited to operate under conditions of uncertainty. However, its use should be avoided in environments where the SNR may fall to $10 \mathrm{~dB}$, in which case the accuracy of the decision drops significantly.

The percentages of effectiveness shown in Table 2 indicate the level of certainty in the decision made by the Network of Networks regarding the belonging of the K shape parameter to a reduced interval. For example, the level of success of the Network of Networks trained with $20 \mathrm{~dB}$ when facing groups of samples with $30 \mathrm{~dB}$ is $91.6 \%$. This means that, as average, every 100 identifications at least 91 commit less than 0.392 units of error, which corresponds to a $4 \%$ of the range of initial uncertainty of 9.75 (that is half of the range of $v$ values for sea clutter that goes from 0.1 to 19.6). However, this 91.6 percent can be raised if the final decision interval is slightly sacrificed. To understand this, an analysis is needed on mistakes committed by each Network of Networks. 
The next sub-section shows the analysis for the case of the previously addressed network trained for $20 \mathrm{~dB}$ of SNR and working with clutter samples with $30 \mathrm{~dB}$ of SNR.

\subsection{Networks mistakes characterization}

It is important to study the distribution of the errors committed by a neural network or any estimation system in general. Table 2 quantifies the amount of errors but not their magnitude, presenting thus an incomplete analysis. The distribution of the mistakes committed by the Network of Networks trained with $20 \mathrm{~dB}$ of SNR when exposed to clutter samples with $30 \mathrm{~dB}$ of SNR (its worse performance) can be seen in Fig. 4 which plots a confusion matrix. Note that the matrix is divided into two parts due to its large size.
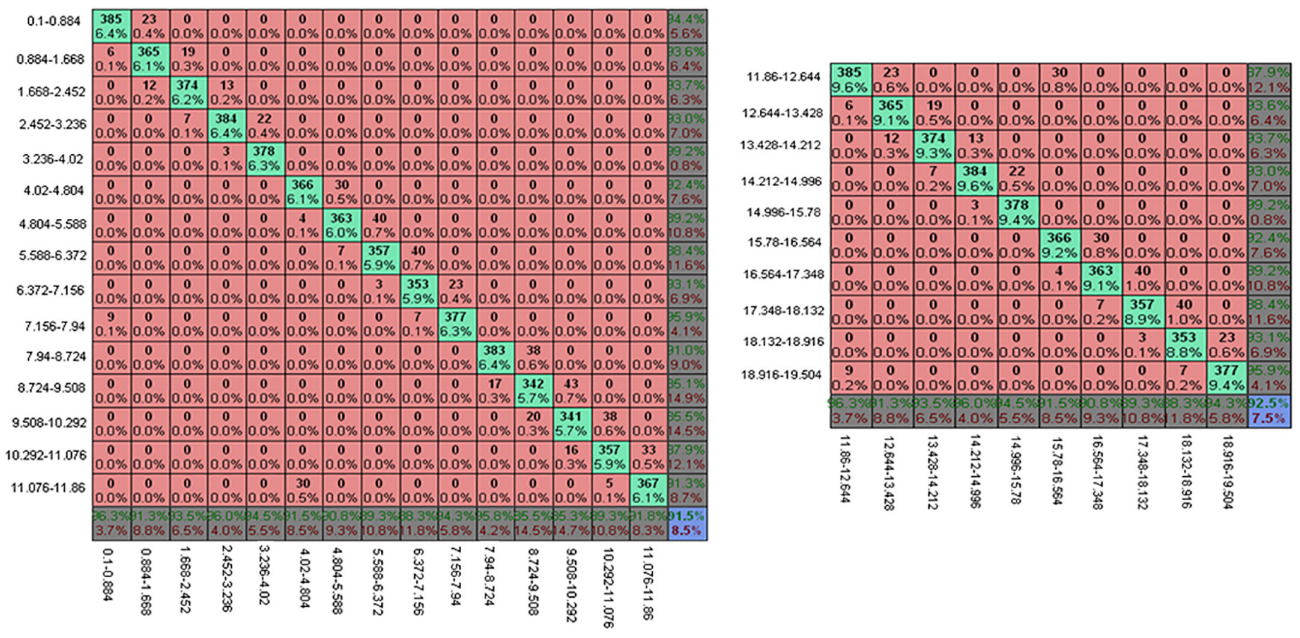

Figure 4. Representation of Committed Errors through a Confusion Matrix.

Each row of the matrix stands for the correct response known in advance by the user in the role of trainer. On the contrary, the columns provide the decision made by the Network of Networks. So, it can be understood that the green squares from the diagonal identify favorable results for cases in which the network made the correct choice.

The reader will notice that most of the errors committed by the Network of Networks are accumulated around the correct answers. The fact constitutes a very advantageous outcome as it means that a remarkable improve can be obtained on the percentage of correct answers by allowing a slight increase in the magnitude of error. Hence, the accuracy will reach $98 \%$ with only an increase of $2 \%$ in the error. Additionally, if another increase of $2 \%$ is allowed the hits will reach $99 \%$. The latter represents the achievement of an effective identification in a range of 0.784 ( $8 \%$ of the initial uncertainty). 


\section{CONCLUSIONS}

An effective solution was given to the identification of the $\mathrm{K}$ distribution shape parameter with the a priori knowledge of the scale parameter. After failing in several attempts to solve the presented problem with a single neural network, the authors proceeded to create a Network of Networks that achieved the estimation with a high percentage of success (99\%). In an innovative solution, the Network of Networks was composed by several neural networks trained independently in order to solve fractions of the main problem. As a training base, 200 samples were taken from histograms of the $\mathrm{K}$ distribution to describe the problem to the network.

\section{REFERENCES}

1. Barton, D. K., Leonov, S. A. Radar Technology Encyclopedia. Massachusetts; Artech House. 1998.

2. Cowper, M. Nonlinear processing of non-Gaussian stochastic and chaotic deterministic time series. Edinburgh. The University of Edinburgh. 2000.

3. Dong, Y. Distribution of X-Band High Resolution and High Grazing Angle Sea Clutter. Defence Science and Technology Organisation. 2006:2-50.

4. Ishii, S., Okamoto, Y., Utsunomiya, T. Study on Effect of Sea Condition for Statistical Properties of Sea Clutter using an X-band Radar. IEEJ Transactions on Fundamentals and Materials. 2011; 131(1):263-69.

5. Ishii, S., Sayama, S., Mizutani, K. Effect of Changes in Sea-Surface State on Statistical Characteristics of Sea Clutter with X-band Radar. Wireless Engineering and Technology. 2011; 2(3):175-83.

6. Totir, F., Rador, E., Anton, L. Advanced Sea Clutter Models and their Usefulness for Target Detection. MTA Review. 2008;18(3):257-72.

7. Yim, J. Z., Chou, C. R., Wong, K. A study of the statistics of sea clutter in the northern coast of Taiwan. Proceedings of the Seventeenth, International Offshore and Polar Engineering conference. 2007, Lisbon, Portugal.

8. Sayama, S., Ishii, S. Suppression of Log-Normal Distributed Weather Clutter Observed by an S-Band Radar. Wireless Engineering and Technology. 2013;4(3):125-33.

9. Oyedokun, O. Sea Clutter Simulation. (Master of Science in Engineering). Cape Town. 2012. 10. Tunaley, J. K. E. K-Distribution Algorithm. 2010. Master Thesis, London Research and Development Corporation, London, UK.

11. Yowchyun, L. Generation of Clutter within a Structured Target Synthesizer. 2012. PhD Thesis, Naval Postgraduate School, Monterey, California, USA.

12. Machado Fernández, J. R., \& Bueno González,A. Clasificación del Clutter Marino utilizando Redes Neuronales Artificiales. Cuba. Instituto Superior Politécnico José Antonio Echeverría (ISPJAE). 2012.

13. Antipov, I. Simulation of Sea Clutter Returns. DSTO Electronic and Surveillance Research Laboratory. 1998:2-5.

14. Haykin, S., Bakker, R., Currie, B. W. Uncovering Nonlinear Dynamics-The Case Study of Sea Clutter. Proceedings of the IEEE. 2002; 90(5):860-81.

15. Ward, K., Tough, R., Watts, S. Sea Clutter, the K Distribution and Radar Performance, 2nd Edition: Institution of Engineering and Technology, UK. 2013.

16. Bacallao Vidal, J. C. Un modelo Teórico de la Técnica DRACEC. Metodología del Proceso de Adaptación al Fondo. PhD Thesis, Instituto Técnico Militar "José Martî", La Habana, Cuba. 2003 
17. Mostafa Saleh, N. Automated Oil Spill Detection with Ship Borne Radar. 2004.

18. Miglicaccio, M., Gambardella, A., Tranfaglia, M. Oil Spill Observation by Means of Polarimetric SAR data. Proceedings of SEASAR. 2006:23-6.

19. Brekke, E., Hallingstad, O., Glattetre, J. Target Tracking in Heavy-Tailed Clutter Using Amplitude Information. 12th International Conference on Information Fusion Seattle, WA, USA, July 6-9, 2009.

20. Haykin, S., Puthusserypady, S. Chaotic Dynamics of Sea Clutter. Chaos: An Interdisciplinary Journal of Nonlinear Science. 1997; 7(4):777-802.

21. O'Connor, A. N. Probability Distributions Used in Reliability Engineering. Maryland: University of Maryland. 2011.

22. Kriesel, D. A Brief Introduction to Neural Networks. 1st Edition: Springer, USA. 2005.

23. Ahmad Zuri, M. Radar Performance Analysis in the presence of Sea Clutter. IEEE Transactions on Aerospace and Electronic Systems. 2005; 36(4):1429-439.

24. Mezache, A., Sahed, M. Parameter Estimation in K-Distributed Clutter with Noise using Nonlinear Networks. Master Thesis, Université de Constantine, Faculté des Sciences de l'Ingénieur. 2010 .

25. Serrano, J. D., Martín, Emiliano. Redes Neuronales Artificiales: Universidad de Valencia. 2009. 26. Mark Hudson, B., Hagan, M. T., Demuth, H. B.. Neural Network Toolbox ${ }^{\text {TM }}$ User's Guide. Mathworks 2011.

27. Garzón Guerrero, J. A. Clasificación de blancos de radar en ambientes de ruido arbitrario mediante resonancias naturales y técnicas de componentes principales. PhD Thesis, Universidad de Granada, 2012.

28. Kuck, M. Constant False Alarm Rate Detection of Radar Signals with Artificial Neural Networks. Bachelor Thesis. 1996.

29. Lee, J. H., Kim, H. T. Radar Target Discrimination Using Neural Networks. Aerospace and Electronics. 2010:358-60.

30. Mezzoug, K., Djebbar, B. Étude Comparative d'un Détecteur CFAR Neuronal de Plusieurs Cibles Radar dans un Fouillis de type K-Distribution. Technical Report, Informatics Department, Sciences Faculty, USTMB-Oran, Algeria, 2008.

31. Munro, D. J., Ersoy, O. K. A Neural Network Approach to Stationary Target. Technical Report, 1993.

32. Principe, J. C., Kin, M. Target Prescreening Based on a Quadratic Gamma Discriminator. IEEE Transactions on Aerospace and Electronic Systems. 1998;34(3):706-15.

33. Vicen Bueno, R., Carrasco Álvarez, R., Rosa Zurera, M., Nieto Borge, J. C. Sea Clutter Reduction and Target Enhancement by Neural Networks in a Marine Radar System. Sensors journal. 2009; 9(3):1913-36.

34. Vicen Bueno, R., Jarabo Amores, M. P., Rosa Zurera, M., Mata Moya, D., \& Gil Pita, R. Robustness with respect to the signal-to-noise ratio of MLP-Based Detectors in Weibull clutter. 15th European Signal Processing Conference, 2007:1736-40.

35. Vicen Bueno, R., Rosa Zurera, M., Jarabo Amores, M. P., de la Mata Moya, D. Coherent Detection of Swerling 0 Targets in Sea-Ice Weibull-Distributed Clutter Using Neural Networks. IEEE Transactions on Instrumentation and Measurement. 2010; 59(12):3139-51.

36. Vicen Bueno, R., Rosa Zurera, M., Jarabo Amores, M. P., \& Gil Pita, R. Automatic target detection in simulated ground clutter (Weibull distributed) by multilayer perceptrons in a lowresolution coherent radar. IET Radar, Sonar and Navigation. 2009;4(2):315-28.

37. Zhu Yong, S. W., Jiangmin Qin, Wu Han. New Method of Radar Target Recognition Based on Neural Network. Proceedings of ICSP'96. 1996. 